\title{
THE HIPPOCAMPUS IN MULTIPLE SCLEROSIS
}

${ }^{1,2}$ Maria A. Rocca, MD, ${ }^{3,4}$ Prof. Frederik Barkhof, MD, PhD, ${ }^{5}$ Prof. John De Luca, PhD, ${ }^{6}$ Prof.

Jonas Frisen, MD, PhD, ${ }^{7}$ Prof. Jeroen J.G. Geurts, MSc, PhD, ${ }^{7}$ Hanneke E. Hulst, MSc, PhD,

${ }^{8}$ Jaume Sastre-Garriga, MD, PhD, ${ }^{1,2}$ Prof. Massimo Filippi, MD, for the MAGNIMS Study Group*

${ }^{1}$ Neuroimaging Research Unit, and ${ }^{2}$ Department of Neurology, Institute of Experimental Neurology, San Raffaele Scientific Institute, Vita-Salute San Raffaele University, Milan, Italy; ${ }^{3}$ Department of Radiology and Nuclear Medicine, MS Center Amsterdam, VU University Medical Center, Amsterdam, The Netherlands; ${ }^{4}$ Institutes of Neurology and Healthcare Engineering, UCL Institute of Neurology, London, UK; ${ }^{5}$ Kessler Foundation, West Orange, New Jersey; Department of Physical Medicine and Rehabilitation, Rutgers New Jersey Medical School, Newark, New Jersey; ${ }^{6}$ Department of Cell and Molecular Biology, Karolinska Institute, Sweden; ${ }^{7}$ Department of Anatomy \& Neurosciences, VU University Medical Center, VUmc MS Center Amsterdam, Amsterdam Neuroscience, Amsterdam, The Netherlands; ${ }^{8}$ Department of Neurology/Neuroimmunology, Multiple Sclerosis Centre of Catalonia (Cemcat), Hospital Universitari Vall d'Hebron, Universitat Autònoma de Barcelona, Barcelona, Spain.

*See the Appendix for the list of MAGNIMS Study Group members.

Type of manuscript: personal view.

Word count: summary: 170, main text: 3989 .

Number of figures: 3. Appendix: 2 Panels, 1 Table, 2 Figures. Number of references: 81.

Correspondence to: Prof. Massimo Filippi, Neuroimaging Research Unit, Institute of Experimental Neurology, Division of Neuroscience, San Raffaele Scientific Institute, Vita-Salute San Raffaele University, Via Olgettina, 60, 20132 Milan, Italy. Telephone number: \#39-02-26433033; Fax number: \#39-02-2643-5972; E mail address: filippi.massimo@ @sr.it 


\section{Summary}

Some of the clinical manifestations of multiple sclerosis (MS), such as memory impairment and depression, are, at least partially, related to involvement of the hippocampus. Pathological studies have demonstrated extensive demyelination, neuronal damage and synaptic abnormalities in the hippocampus of MS patients. Improvements in MRI technology have provided novel ways to assess, in vivo, hippocampal involvement. It is now accepted that clinical manifestations related to the hippocampi are due not only to focal hippocampal damage, but also to disconnection of the hippocampus from several brain networks.

There is evidence of anatomical and functional subspecialization of the different hippocampal subfields, which result in regional variability in susceptibility to both damaging and reparative mechanisms following damage occurrence. The hippocampus also has an important role in plasticity and neurogenesis, both of which have the potential to contribute to functional preservation and restoration. All of this underlines the importance of an evaluation of the hippocampus not only for improving our understanding of MS clinical manifestations, but also as a potential future target for treatment. 


\section{Introduction}

Extensive demyelination, neuronal damage and synaptic abnormalities are the characteristic pathological features of hippocampal involvement in many people with multiple sclerosis (MS). Advances in magnetic resonance imaging (MRI) techniques have provided several in vivo tools to detect hippocampal damage and investigate its clinical relevance in several neurological conditions. The application of these methods in MS has helped to highlight that not only focal hippocampal involvement, in terms of lesions, microstructural abnormalities and tissue loss, but also structural and functional disconnections of the hippocampus from several brain networks contribute, at least in part, to explain some of the clinical deficits of these patients, such as cognitive impairment and depression.

Pathological and MRI studies have shown that different hippocampal subfields have different susceptibility to damage. In addition to damaging processes, there is also evidence of an in vivo expansion of the hippocampal dentate gyrus (DG), that may represent a compensatory response to central nervous system (CNS) inflammation.

We discuss the current state of the knowledge concerning hippocampal involvement in MS. Symptoms ascribable to hippocampal dysfunction and the pathological substrates of this involvement are considered. We then summarize the literature where MRI has been applied to provide in vivo measures of hippocampal damage and dysfunction. Next, evidence of plasticity in this structure is examined in order to give a base to the emerging view of the hippocampus as a candidate target for treatment strategies.

\section{Clinical manifestations of hippocampal damage}

Current knowledge of hippocampal function in humans derives from seminal descriptions of single patients with lesions affecting this region. Recent studies have used MRI to define hippocampal morphology and connectivity, linking these with clinical and behavioral assessments. 
Figure 1 provides a schematic representation of the main hippocampal subdivisions and connections. Appendix presents some evidences supporting hippocampal functional specialization.

An event-related functional MRI study of encoding and retrieval in ten healthy individuals have shown that distinct hippocampal subregions are differentially involved in memory function, with the dentate gyrus (DG) (DG) and the cornus ammonis (CA) fields 2 and 3 (CA2 and 3) selectively recruited during episodic memory formation and the subiculum activated during retrieval processes. ${ }^{1}$ Consistent with this regional specialization, cell loss in the DG, CA3 and CA4 assessed on surgical resection of 100 consecutive epileptic patients correlated with deficits in declarative memory formation, evaluated from intracarotid amobarbital testing and non-invasive verbal memory assessment before surgery. ${ }^{2}$

Moving to MS, numerous studies have shown that MS patients suffer primarily from deficient initial acquisition of learning rather than impairments in retrieval from long term storage, as assessed using list-learning tasks. ${ }^{3,4}$ Deficits were specific to learning and memory rather than other aspects of cognition (e.g., processing speed and working memory). Memory impairment occurs from the early stages of the disease, in patients with clinically isolated syndromes (CIS). ${ }^{5}$ Recently, decreased performance in a pattern separation task was demonstrated in a cross-sectional study of 19 patients with early relapsing-remitting (RR) MS (within 6-to-18 months after a first neurological episode suggestive of MS), in comparison to healthy controls matched for age, gender and education level, in the absence of information processing speed (evaluated with the Computerized Speed Cognitive Test ) and visuospatial memory (evaluated with the Brief Visuospatial Memory Test) impairment. ${ }^{6}$ Since it has been shown a critical role of the DG in pattern separation, ${ }^{7}$ impaired performance in this task could be seen as evidence that brain areas implicated in pattern separation, for which the DG is crucial, are involved in early MS.

Depression has also been associated with hippocampal involvement in MS and affects up to $50 \%$ of patients. ${ }^{8}$ Many mechanisms have been implicated in the pathogenesis of depression in MS, including genetic, biochemical, immunological and psychosocial factors. However, abnormalities 
detected using MRI, including gadolinium-enhancing lesions, ${ }^{9} \mathrm{~T} 2$ lesions in strategic white matter (WM) regions, atrophy of specific grey matter (GM) structures and abnormal recruitment of specific pathways, seem to play a prominent role. ${ }^{8}$ For this review, only those mechanisms that may be related to hippocampal involvement will be discussed. Some of the research on the link between hippocampal damage and depression in MS has been inspired by studies in patients with major depression, in whom hippocampal atrophy is present from the first episode of the disease, a shown by a random-effect meta-analysis of MRI studies. ${ }^{10}$ Several candidate mechanisms for hippocampal atrophy in depressed patients have been examined, some of which are based on the assumption that depression is a stress-related disorder. ${ }^{11}$ Disturbed hypothalamic pituitary adrenal (HPA) axis function is a feature of depression, and it has been proposed that adrenal hypersecretion of glucocorticoids, in particular cortisol, could cause hippocampal atrophy. ${ }^{11}$ Interestingly, there is evidence of increased HPA axis activity in a large proportion of MS patients (53 of 86 patients, $62 \%$ ) particularly in those with progressive MS. ${ }^{12}$

Psychosis has also been related to hippocampal involvement in psychiatric patients (i.e., schizophrenia). ${ }^{13}$ Unfortunately, as discussed in a recent epidemiological study, ${ }^{14}$ the frequency of psychosis in MS is currently underestimated, due to heterogeneity between studies in data sources, populations, and definitions of psychiatric comorbidity. As a consequence, further investigations are needed to explore this manifestation and its underlying substrates.

\section{Pathological basis of hippocampal damage in MS}

Hippocampal demyelination in MS is common and extensive. ${ }^{15-18}$ A study of 19 chronic MS cases and 7 control samples with no neurologic or psychiatric diseases showed demyelinated lesions in $15(79 \%)$ patients and none of controls. Mixed intrahippocampal-perihippocampal lesions were large and did not respect anatomical borders. These lesions were found in six of seven MS cases with cognitive impairment (extrapolated from clinical files of tissue donors) and three of ten cases without cognitive impairment. Small, isolated intrahippocampal lesions were also frequently seen in 
MS patients, and had a specific anatomical predilection, with consistent sparing of both the CA2 subregion and the hilus of the DG in all MS cases (Figure 2). ${ }^{16}$ Another investigation found demyelinated hippocampal lesions in 24 of 45 (53\%) progressive MS cases, with a mean of 30.4\% $(\mathrm{SD}=22 \%$, range $=1.8 \%-95.4 \%)$ of demyelination of total hippocampal cross-sectional area. ${ }^{18}$ The majority of lesions were chronic and located subpially. Significant reductions of neuron counts in comparison to controls were detected in CA1 (percentage difference: $-27.0 \%$ ) and CA3-2 (percentage difference: $-29.7 \%$ ), with no difference between patients with and those without demyelinated lesions. In another study of 22 MS patients and 9 control patients, hippocampal demyelination was extensive in 12 patients and absent or minimal in the remaining ten patients. Compared to control hippocampi, no clear neuronal loss was detected in demyelinated hippocampi, whereas a significant decrease of synaptic density was found, indicating that myelin loss can induce synaptic loss in hippocampal neurons. Demyelinated hippocampi had a decreased expression of proteins involved in axonal transport, synaptic plasticity, memory, learning and neuronal survival. ${ }^{17}$ To identify the mechanisms underlying the regulation of neuronal gene expression, microRNA (miRNAs) were compared between myelinated and demyelinated hippocampi. ${ }^{19}$ An up-regulation of several neuronal miRNAs, including miR-124, and decreased expression of neuronal genes, including AMPA receptors, were found with hippocampal demyelination. Significant reductions in the number of synapses associated with hippocampal neurons were found in demyelinated regions. ${ }^{17,20}$ The cause of this synaptic loss is not clear, but may be partly attributable to 'synaptic stripping' by microglia. ${ }^{21}$ Inflammatory demyelination may lead to altered AMPA receptor expression $^{17,19}$ as well as a selective loss of interneurons and subsequent disruption of the GABAergic system. In a presumed attempt to 'rescue' the micro-environment from excitotoxicity by excess glutamate, microglia are thought to strip the neurons from their synapses, by displacing presynaptic terminals. ${ }^{21}$ Flagging of synapses for subsequent stripping may occur through activation of the complement system. ${ }^{20}$ Microglia are regionally present in microscopically investigated hippocampal specimens, ${ }^{16,17}$ mostly in those areas 'at risk' of being demyelinated. This is in 
contrast with already demyelinated areas, which are largely devoid of microglial activity and macrophages. ${ }^{16}$ Activated microglia and macrophages upregulate expression of the $18 \mathrm{kDa}$ translocator protein (TSPO), which can be imaged in vivo by positron emission tomography (PET) TSPO radioligands. A combined PET and 7T MRI study found significant microglial and macrophage activation in several GM structures, including the hippocampus, in RRMS $(\mathrm{n}=12)$ and secondary progressive (SP) MS ( $\mathrm{n}=15)$ patients, even in absence of focal lesions visuallyidentifiable at ultra-high field. ${ }^{22}$

GM inflammation is a hot topic in current MS research, although how far (adaptive) immunological processes are causal to demyelination and subsequent neuronal and synaptic changes in the temporal chain of pathological events, is far from resolved. Epigenetic mechanisms (e.g., DNA methylation) have been shown to alter gene expression in the hippocampus of MS patients. ${ }^{23}$ Whether these mechanisms are involved in the pathogenesis or represent a secondary consequence of demyelination remains to be defined.

\section{Imaging hippocampal damage in MS using MRI}

Considerable effort has been spent on improving MR imaging of the hippocampus in MS, by applying methods that are currently used to assess other CNS structures and neurological conditions, in which the hippocampus is among the primary target regions (e.g., dementias). This has been motived on the one hand by an attempt to explain clinical symptoms that may be directly or indirectly related to hippocampal involvement, and on the other by the goal of improved understanding of some of the physiopathological processes going on in the disease (for example, atrophy development). Appendix summarises the main MRI studies in MS that have included the hippocampus as a target of investigation by assessing lesions, atrophy as well as structural and functional connectivity abnormalities at this level. As can be seen, hippocampal atrophy is the most frequently assessed MRI measure, with quite heterogeneous findings, likely because of differences 
in demographic and clinical characteristics of the subjects enrolled, as well as methodological issues, as summarised in the Appendix.

Lesions. As is the case for other GM regions, conventional T2-weighted imaging is suboptimal for demonstrating in vivo the presence of focal hippocampal lesions. The use of specialised imaging techniques, such as double inversion recovery (DIR), has allowed the detection of about 50\% more lesions in comparison to conventional T2-weighted scans (Figure 3). ${ }^{24}$ However, the poor signal-to-noise ratio and spatial resolution of DIR images preclude the identification of subfield involvement achieved with pathological assessment; combined MRIpathology studies of hippocampal involvement are currently completely lacking. A three-year clinical and MRI study showed that the number of hippocampal lesions is relatively stable over time in 13 MS patients (9 with RRMS, 4 with SPMS). ${ }^{25}$ Despite this, these lesions are clinically relevant, since they are associated with deficits in visuo-spatial memory ${ }^{25}$ and epilepsy. ${ }^{26}$

One way to circumvent the limited spatial resolution is to use quantitative MR techniques to estimate the overall burden of microscopic hippocampal involvement. Using diffusion tensor (DT) MRI, it has been demonstrated that microstructural hippocampal abnormalities occur relatively early in the course of the disease, in the CIS phase ( $n=37$ CIS studied within 6 months from the first clinical attack), even though they are more pronounced in RRMS ( $=32) .{ }^{27}$ Increased hippocampal water diffusivity in CIS patients was correlated with episodic verbal memory performance, assessed with the California Verbal Learning Test, and helped to distinguish memory-impaired from memory-preserved CIS patients at the delayed recall Selective Reminding Tests, suggesting that it might represent an early biomarker for monitoring hippocampus-related memory impairment.

Atrophy. Hippocampal atrophy is likely to result both from local pathological processes and from secondary retrograde phenomena due to damage in tracts and regions connected to the hippocampus. ${ }^{28}$ Current MRI studies of atrophy (Appendix) have all excluded patients with relapses or steroids treatment in the month prior to MRI to minimise their influence on volumetric assessment. Hippocampal atrophy in MS has been documented in many studies, using different 
methods of analysis (Appendix), and has been correlated with both global cognitive impairment and impairment in specific cognitive tests, mostly in the domain of memory. ${ }^{29-33}$ Hippocampal atrophy is present both in RRMS (including pediatric patients) ${ }^{33}$ and progressive MS patients, suggesting that it might occur early in the course of the disease (Online Figure 1). ${ }^{31,34,35}$ A study that examined the role of patients' age on brain GM volumetric abnormalities found significant excess atrophy of the hippocampus in younger-onset (mean age=30.4 years, $\mathrm{SD}=3.2$ years) versus olderonset (mean age $=48.7$ years, $\mathrm{SD}=3.3$ years), whereas for all other $\mathrm{GM}$ structures, atrophy was similar in the two groups, suggesting severe tissue abnormalities in hippocampal substructures in people with earlier onset disease. ${ }^{36}$

In line with other neurological conditions ${ }^{37,38}$ and with pathology studies in MS, ${ }^{16,17}$ studies that have applied regional methods of analysis have suggested that hippocampal subregions have different vulnerability to damage, with CA1 and the subiculum as the most severely damaged regions. ${ }^{35,39,40}$ Poor performance in hippocampal-related functions, such as visuospatial memory, verbal memory and memory acquisition correlated with atrophy of the previous regions. ${ }^{35,39} \mathrm{~A}$ recent longitudinal study in 56 CIS patients found early atrophy of the CA4/DG subfield, which spread to the $\mathrm{CA} 1$ after one year. ${ }^{40} \mathrm{CA} 1$ atrophy helped to explain verbal memory performance deficits, assessed with the Selective Reminding Test, at this timepoint. ${ }^{40}$

Several MRI studies have shown hippocampal atrophy in MS patients with depressive symptoms (Appendix). ${ }^{41-43}$ Patients with RRMS ( $\left.\mathrm{n}=29\right)$ and depression, assessed using the Beck depression scale, had a preferential atrophy of the CA23 and DG regions, which correlated with higher cortisol levels, ${ }^{42}$ consistently with the vulnerability of these regions to the effects of elevated endogenous glucocorticoids.

An enlargement of part of the DG has been detected in vivo using radial mapping techniques. ${ }^{44}$ Interestingly, DG enlargement was more pronounced in RRMS $(n=28)$ compared to SPMS ( $n=34)$ patients and correlated with brain T2-hyperintense lesion volume. In RRMS, DG enlargement correlated with better performance at hippocampal verbal memory tests, whereas in 
SPMS it correlated with poor cognitive performance, suggesting that there may be a reactive process induced by inflammatory lesions aimed at rescuing the functional competence of hippocampal circuitry (Online Figure2). ${ }^{44}$ Clearly, these results need to be replicated and since there is no canonical method for measuring hippocampal subregional volumes in vivo, they should be interpreted with caution.

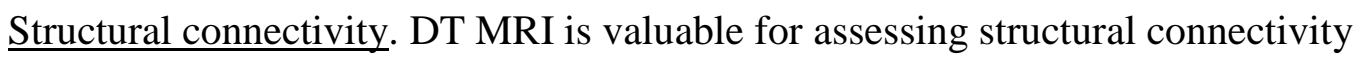
abnormalities within the hippocampal network. Consistent alterations of DT MRI derived indexes of WM tracts connecting the hippocampus to other GM regions (i.e., decreased fractional anisotropy and increased mean diffusivity) indicating reduced connectivity have been demonstrated by several studies in MS patients with different disease clinical phenotypes (Appendix) and have been correlated with memory impairment. ${ }^{34}$

fMRI abnormalities. Hippocampal involvement in MS has also been demonstrated using fMRI techniques, both with hippocampus-related active fMRI tasks and by analyzing resting state (RS) functional connectivity (FC). ${ }^{45-48}$ Different patterns of activation (increased activation, decreased activation, or more recently, increased deactivation) ${ }^{49}$ and FC (increased RS FC or decreased RS FC) have been shown in patients with and without cognitive deficits or depression (Appendix). ${ }^{50,51}$

These fMRI abnormalities are seen prior to the development of atrophy and hippocampusrelated cognitive deficits, suggesting that they may be an early indicator of hippocampal dysfunction. ${ }^{45-47}$

\section{Plasticity of the hippocampal network}

The behavioral and neuroimaging effects of (aerobic) exercise, cognitive rehabilitation, and more specifically memory retraining, in relation to the hippocampal memory network has been extensively studied in several populations, such as healthy aging, mild cognitive impairment, psychiatric disorders, Alzheimer's disease and to a lesser extent MS. ${ }^{52-56}$ Results are rather 
inconclusive, due to methodological differences and often small sample sizes. However, they do point towards beneficial effects of interventions on hippocampal memory function, hippocampal volume and hippocampal activity.

Several mechanisms have been implicated in hippocampal structure changes, including trophic factors (insulin-like growth factor 1 and brain-derived neurotrophic factor), angiogenesis and neurogenesis. It has been challenging to assess the extent of postnatal neurogenesis in humans, since the methods used in experimental animals that provide clear-cut results are not applicable in humans. However, several lines of evidence support continuous neurogenesis in the human DG. A seminal study assessed the incorporation of the thymidine analogue $\mathrm{BrdU}$ in five cancer patients (squamous cell carcinomas of the tongue, larynx or pharynx) who had received this compound for diagnostic purposes, and provided the first evidence for adult neurogenesis in humans ${ }^{57}$ In another approach, ${ }^{14} \mathrm{C}$ from nuclear bomb tests was measured in genomic DNA of hippocampal neurons, and demonstrated continuous hippocampal neurogenesis throughout adulthood ${ }^{58}$ The process of adult hippocampal neurogenesis provides the DG with neurons with unique electrophysiological properties. These new neurons are hyperexcitable for about a month after their formation. ${ }^{59}$ Although at any given time they constitute a small proportion of the DG neurons, their special properties in a population of otherwise largely inhibited neurons, gives them substantial influence on the plasticity of the circuitry. Although the impact of neurogenesis on hippocampal function has been challenged ${ }^{60}$ it has been demonstrated that hippocampal memory formation is positively correlated with neurogenesis. ${ }^{61}$ One recent study on brain tissues from 28 deceased subjects between 14 and 79 years of age ${ }^{62}$ confirmed that human hippocampal neurogenesis occurs throughout aging in healthy subjects with no reported cognitive impairment and no neurological or psychiatric diagnoses during life, whereas another study found a sharp decline during childhood of DG neurogenesis,${ }^{63}$ thus challenging the notion that adult neurogenesis occurs in the human hippocampus. Methodological and conceptual factors that could explain these opposing results have been critically discussed in a recent review, ${ }^{64}$ but this is beyond our remit here. 
A recent meta-analysis of 14 controlled trials examined the effects of aerobic exercise on hippocampal volume in humans (i.e., a mix of older adults aged between 65 and 76 years, patients with mild cognitive impairment, Alzheimer's disease, and psychiatric disorders).$^{52}$ Aerobic exercise had no effect on total hippocampal volume across the 737 participants, while it contributed to volume preservation over time of the left hippocampus, when the two hippocampi were analyzed separately. As discussed by these authors, ${ }^{52}$ more high-quality studies, possibly with longer followup duration (more than 12 months) are now needed to disentangle the effects of aerobic exercise on hippocampal volume and to ascertain the possibility (and basis) of a laterality effect.

A study in healthy elderly people $(n=26$, mean age $=67.9$ years, $S D=3.3$ years $)$ demonstrated increases in hippocampal volume after intensive training (twice a week for 6 months, followed by once a week for 12 months; 90 minutes training). ${ }^{65}$ The hippocampal changes were seen in people participating in fitness sport and dance; however, the changes were more pronounced and widespread in the dance intervention, including the right subiculum and left DG. Future studies should investigate whether such 'combination studies' might exert larger effects on the hippocampal network than single modality studies. Also, intensity and duration of the intervention might determine whether structural changes in the hippocampus can be observed. Functional changes in hippocampal activity, contrary to volume changes, can occur relatively fast (already seen after two weeks of cognitive training) and were even observed in patients that already had deficits in the memory domain (12 patients with mild cognitive impairment according to recommendations from an International Committee). ${ }^{66}$ These changes in hippocampal activation were associated with improvement at verbal memory, engendering a restoration of the deficient activity. ${ }^{66}$

\section{A potential future target for treatment}

Evidence for hippocampal plasticity and verbal and visuo-spatial memory deficits suggests that the hippocampus might be an interesting target for cognitive rehabilitation studies aimed at improving memory deficits. Using MRI measures to monitor interventions that particularly aim to 
remediate episodic or visuospatial memory disturbance would seem to be the logical first step. However, functional and structural changes of the hippocampus could also be used as markers of global beneficial effects of non-hippocampus specific (e.g., constraint-induced therapy) or rather general (e.g., aerobic exercise) therapies, as suggested in other conditions. For example, in chronic stroke patients $(\mathrm{n}=16)$, positive effects of constraint-induced therapy (3 hours daily for 10 consecutive weekdays) on motor function were associated with volume increases not only in brain sensory and motor areas, but also in the hippocampus. ${ }^{67}$ In a randomized study of 100 older individuals (mean age $=70.1$ years, $\mathrm{SD}=7$, range $=55-87$ years) with mild cognitive impairment, ${ }^{68}$ subjects randomized to 6 months of computerized cognitive training had no decline in memory performance and enhanced FC between the hippocampus and frontal lobe, whereas subjects randomized to progressive resistance training improved in global cognition and showed an enlargement of their posterior cingulate cortex.

Data on the efficacy of cognitive or motor rehabilitation in patients with MS is scarce, and only a handful of studies have used MRI to monitor functional or structural brain changes (see ${ }^{69}$ for a review). Only one of these specifically examined the effect of memory training in MS patients, using the modified Story Memory Technique. In this double-blind, placebo-controlled, clinical trial, eight MS patients were randomized to treatment and eight to placebo-control. After five weeks of training, patients in the treatment group improved in memory performance and had greater activation during a memory task of several brain regions, including the hippocampus. ${ }^{70} \mathrm{~A}$ subanalysis of RS FC data from seven patients in each group showed increased connectivity between the hippocampi and several cortical regions in the treatment group only. ${ }^{71}$ A further report on just two memory-impaired patients indicated improvement in memory function as well as increase of hippocampal volume and FC in one patient after an aerobic exercise program (30-minute sessions 3 times per week for 3 months), while no changes were detected in the patient undergoing nonaerobic (stretching) exercise. ${ }^{72}$ 
Halting inflammation through the use of disease-modifying treatments might be another strategy to promote hippocampal-related functions. Indeed, inflammatory CNS conditions are able to modulate homeostasis of neural stem and precursor cells (NPCs) and perturb some aspects of neurogenesis. ${ }^{73}$ Acute inflammation has been shown to reduce neurogenesis. ${ }^{74}$ A recent study demonstrated that while the inflammatory environment initially promotes hippocampal NPC proliferation, it also reduces the capacity of these cells to generate mature neurons and, over the long-term, modifies NPC differentiation, giving prominence to gliogenesis. ${ }^{75}$

Interestingly, preliminary data have shown that FTY720 (fingolimod), a sphingosine 1phosphate receptor agonist, is able to increase the viability and neurogenicity of irradiated neural stem cells from the hippocampus. ${ }^{76}$ In a mouse model of MS, the systemic injection of minocycline, a microglial inhibitor, prevented DG neurodegeneration, DTI changes and memory impairment. ${ }^{77}$ Recent data in 38 MS patients (36 RRMS, 2 SPMS) treated with natalizumab over a 36 month period suggest that including measurement of GM and hippocampal volume changes to monitor drug effects helps to improve understanding of the mechanisms responsible for disability progression in these patients, since atrophy of selected GM structures was associated with disability progression over time. ${ }^{78}$

Future research is warranted to clarify the effects of pharmacological treatments on NPC maturation pathways and, ultimately, on hippocampal functions.

\section{Conclusions and future directions}

Understanding of hippocampal involvement in patients with MS has profoundly improved during the past few years. Developments in MRI techniques have provided in vivo strategies to assess damage to this structure and its progression. The effects of hippocampal disconnection from integrated neural networks have also been highlighted. All this has been pivotal in improving our understanding of some of the clinical deficits of MS patients, including memory impairment and 
depression. The utility of these approaches for monitoring these deficits, and for predicting their worsening, is still to be investigated.

Evaluation of the hippocampus in MS and beyond has revealed its peculiar features, including regional vulnerability to damage, elevated synaptic plasticity and neurogenesis potential, which offer promising perspectives when including this structure as a target in the evaluation of treatment effects in MS.

Many challenges remain, which require a strict collaboration between clinicians, neuropsychologists, pathologists and imaging experts. Standardised approaches for the clinical and neuropsychological assessment of hippocampal related manifestations should be fostered. In addition, there is the need to identify tests capable to monitor hippocampal dysfunction over time, which could be applied in the context of treatment monitoring. Correlative studies between pathology and MRI are warranted to further progress knowledge on hippocampal mechanisms of damage and repair and to develop imaging measures specific for these processes. Clearly, standardisation of MRI procedures and methods of analysis is also needed to move the field forward. Effort should also be spent to define imaging biomarkers (single or composite) to track hippocampal related changes. Longitudinal studies as well as studies enrolling larger numbers of subjects in comparison to those currently available, are now needed to explore further this structure. 


\section{Search strategy and selection criteria}

References for this Review were identified through searches of PubMed with the search terms "Hippocampus", "Memory", "Depression", "Cognition", "Cognitive impairment", "Multiple Sclerosis", "Grey Matter", "White matter", "Lesions", "Connectivity”, "Volume”, "Atrophy”, "Diffusion Tensor MRI", "fMRI", "Resting State fMRI"; "Plasticity"; "Dentate gyrus",

"Treatment", and "Neurogenesis" from 1 Jan 1979 until 1 June 2018. Articles were also identified through searches of the authors' own files. Only papers published in English were reviewed. The final reference list was generated on the basis of originality and relevance to the broad scope of this Review.

\section{Authors' contributions}

MAR and MF developed the idea for the review, organized it and framed the structure of this Review. FB, JDL, JF, JJG, HH and JSS summarized different aspects for the review. The complete Review was commented on, revised, and approved by all authors.

Acknowledgements. FB is supported by the NIHR UCLH biomedical research centre.

\section{Conflicts of interest}

M.A. Rocca received speakers honoraria from Biogen Idec, Novartis, Genzyme, Sanofi-Aventis, Teva and Merck Serono and receives research support from the Italian Ministry of Health and Fondazione Italiana Sclerosi Multipla.

F. Barkhof acts as a consultant to Biogen-Idec, Janssen Alzheimer Immunotherapy, BayerSchering, Merck-Serono, Roche, Novartis, Genzyme, and Sanofi-aventis. He has received sponsorship from EU-H2020, NWO, SMSR, EU-FP7, TEVA, Novartis, and Toshiba. He is on the editorial board of Radiology, Brain, Neuroradiology, MSJ, and Neurology.

J. De Luca received speaker honoraria from Sanofi-Genzyme, consultation to Sanofi-Genzyme, research support from Biogen IDEC, the National Multiple Sclerosis Society and Consortium for Multiple Sclerosis Centers.

J. Frisen has no conflicts of interest.

J.J.G. Geurts serves on the editorial boards of MS Journal, BMC Neurology, MS International, and Neurology, the Scientific Advisory Board of the Dutch MS Research Foundation and MS Academia, Merck-Serono, and has served as a consultant for Merck- Serono, Biogen Idec, Novartis, Genzyme, and Teva Pharmaceuticals.

H.E. Hulst has received compensation for consulting services and/or speaking activities from Merck Serono, Genzyme, Novartis, and Roche.

J. Sastre-Garriga has received compensation for serving on scientific advisory boards or on speaker's bureaus from Biogen Idec, Merck Serono, Novartis, Teva Pharmaceutical Industries, and Sanofi-Aventis.

M. Filippi is Editor-in-Chief of the Journal of Neurology; has received compensation for consulting services and/or speaking activities from Biogen Idec, Merck-Serono, Novartis, and Teva Pharmaceutical Industries; and receives research support from Biogen Idec, Teva Pharmaceutical Industries, Novartis, Roche, Italian Ministry of Health, Fondazione Italiana Sclerosi Multipla, and ARiSLA (Fondazione Italiana di Ricerca per la SLA). 


\section{References}

1. Eldridge LL, Engel SA, Zeineh MM, Bookheimer SY, Knowlton BJ. A dissociation of encoding and retrieval processes in the human hippocampus. J Neurosci 2005;25:3280-6.

2. Coras R, Pauli E, Li J, Schwarz M, Rossler K, Buchfelder M, et al. Differential influence of hippocampal subfields to memory formation: insights from patients with temporal lobe epilepsy. Brain 2014;137:1945-57.

3. DeLuca J, Barbieri-Berger S, Johnson SK. The nature of memory impairments in multiple sclerosis: acquisition versus retrieval. J Clin Exp Neuropsychol 1994;16:183-9.

4. Lafosse JM, Mitchell SM, Corboy JR, Filley CM. The nature of verbal memory impairment in multiple sclerosis: a list-learning and meta-analytic study. Journal of the International Neuropsychological Society: JINS 2013;19:995-1008.

5. Feuillet L, Reuter F, Audoin B, Malikova I, Barrau K, Cherif AA, et al. Early cognitive impairment in patients with clinically isolated syndrome suggestive of multiple sclerosis. Mult Scler 2007;13:124-7.

6. Planche V, Ruet A, Charre-Morin J, Deloire M, Brochet B, Tourdias T. Pattern separation performance is decreased in patients with early multiple sclerosis. Brain and behavior 2017;7:e00739.

7. Berron D, Schutze H, Maass A, Cardenas-Blanco A, Kuijf HJ, Kumaran D, et al. Strong Evidence for Pattern Separation in Human Dentate Gyrus. J Neurosci 2016;36:7569-79.

8. Feinstein A, Magalhaes S, Richard JF, Audet B, Moore C. The link between multiple sclerosis and depression. Nat Rev Neurol 2014;10:507-17.

9. Fassbender K, Schmidt R, Mossner R, Kischka U, Kuhnen J, Schwartz A, et al. Mood disorders and dysfunction of the hypothalamic-pituitary-adrenal axis in multiple sclerosis: association with cerebral inflammation. Arch Neurol 1998;55:66-72.

10. Cole J, Costafreda SG, McGuffin P, Fu CH. Hippocampal atrophy in first episode depression: a metaanalysis of magnetic resonance imaging studies. J Affect Disord 2011;134:483-7.

11. Sapolsky RM. Depression, antidepressants, and the shrinking hippocampus. Proc Natl Acad Sci U S A 2001;98:12320-2.

12. Ysrraelit MC, Gaitan MI, Lopez AS, Correale J. Impaired hypothalamic-pituitary-adrenal axis activity in patients with multiple sclerosis. Neurology 2008;71:1948-54.

13. Suddath RL, Christison GW, Torrey EF, Casanova MF, Weinberger DR. Anatomical abnormalities in the brains of monozygotic twins discordant for schizophrenia. N Engl J Med 1990;322:789-94.

14. Marrie RA, Reingold S, Cohen J, Stuve O, Trojano M, Sorensen PS, et al. The incidence and prevalence of psychiatric disorders in multiple sclerosis: a systematic review. Mult Scler 2015;21:305-17.

15. Vercellino M, Plano F, Votta B, Mutani R, Giordana MT, Cavalla P. Grey matter pathology in multiple sclerosis. J Neuropathol Exp Neurol 2005;64:1101-7.

16. Geurts JJ, Bo L, Roosendaal SD, Hazes T, Daniels R, Barkhof F, et al. Extensive hippocampal demyelination in multiple sclerosis. J Neuropathol Exp Neurol 2007;66:819-27.

17. Dutta R, Chang A, Doud MK, Kidd GJ, Ribaudo MV, Young EA, et al. Demyelination causes synaptic alterations in hippocampi from multiple sclerosis patients. Ann Neurol 2011;69:445-54.

18. Papadopoulos D, Dukes S, Patel R, Nicholas R, Vora A, Reynolds R. Substantial archaeocortical atrophy and neuronal loss in multiple sclerosis. Brain Pathol 2009;19:238-53.

19. Dutta R, Chomyk AM, Chang A, Ribaudo MV, Deckard SA, Doud MK, et al. Hippocampal demyelination and memory dysfunction are associated with increased levels of the neuronal microRNA miR-124 and reduced AMPA receptors. Ann Neurol 2013;73:637-45.

20. Michailidou I, Willems JG, Kooi EJ, van Eden C, Gold SM, Geurts JJ, et al. Complement C1q-C3associated synaptic changes in multiple sclerosis hippocampus. Ann Neurol 2015;77:1007-26.

21. Trapp BD, Wujek JR, Criste GA, Jalabi W, Yin X, Kidd GJ, et al. Evidence for synaptic stripping by cortical microglia. Glia 2007;55:360-8.

22. Herranz E, Gianni C, Louapre C, Treaba CA, Govindarajan ST, Ouellette R, et al. Neuroinflammatory component of gray matter pathology in multiple sclerosis. Ann Neurol 2016;80:776-90.

23. Chomyk AM, Volsko C, Tripathi A, Deckard SA, Trapp BD, Fox RJ, et al. DNA methylation in demyelinated multiple sclerosis hippocampus. Scientific reports 2017;7:8696. 
24. Roosendaal SD, Moraal B, Vrenken H, Castelijns JA, Pouwels PJ, Barkhof F, et al. In vivo MR imaging of hippocampal lesions in multiple sclerosis. J Magn Reson Imaging 2008;27:726-31.

25. Roosendaal SD, Moraal B, Pouwels PJ, Vrenken H, Castelijns JA, Barkhof F, et al. Accumulation of cortical lesions in MS: relation with cognitive impairment. Mult Scler 2009;15:708-14.

26. Calabrese M, Castellaro M, Bertoldo A, De Luca A, Pizzini FB, Ricciardi GK, et al. Epilepsy in multiple sclerosis: The role of temporal lobe damage. Mult Scler 2017;23:473-82.

27. Planche V, Ruet A, Coupe P, Lamargue-Hamel D, Deloire M, Pereira B, et al. Hippocampal microstructural damage correlates with memory impairment in clinically isolated syndrome suggestive of multiple sclerosis. Mult Scler 2017;23:1214-24.

28. Koenig KA, Sakaie KE, Lowe MJ, Lin J, Stone L, Bermel RA, et al. Hippocampal volume is related to cognitive decline and fornicial diffusion measures in multiple sclerosis. Magn Reson Imaging 2014;32:354-8. 29. Damjanovic D, Valsasina P, Rocca MA, Stromillo ML, Gallo A, Enzinger C, et al. Hippocampal and Deep Gray Matter Nuclei Atrophy Is Relevant for Explaining Cognitive Impairment in MS: A Multicenter Study. AJNR Am J Neuroradiol 2017;38:18-24.

30. Preziosa P, Rocca MA, Pagani E, Stromillo ML, Enzinger C, Gallo A, et al. Structural MRI correlates of cognitive impairment in patients with multiple sclerosis: A Multicenter Study. Hum Brain Mapp 2016;37:1627-44.

31. Anderson VM, Fisniku LK, Khaleeli Z, Summers MM, Penny SA, Altmann DR, et al. Hippocampal atrophy in relapsing-remitting and primary progressive MS: a comparative study. Mult Scler 2010;16:108390.

32. Sacco R, Bisecco A, Corbo D, Della Corte M, d'Ambrosio A, Docimo R, et al. Cognitive impairment and memory disorders in relapsing-remitting multiple sclerosis: the role of white matter, gray matter and hippocampus. J Neurol 2015;262:1691-7.

33. Rocca MA, Morelli ME, Amato MP, Moiola L, Ghezzi A, Veggiotti P, et al. Regional hippocampal involvement and cognitive impairment in pediatric multiple sclerosis. Mult Scler 2016;22:628-40.

34. Pardini M, Bergamino M, Bommarito G, Bonzano L, Luigi Mancardi G, Roccatagliata L. Structural correlates of subjective and objective memory performance in multiple sclerosis. Hippocampus 2014;24:436-45.

35. Sicotte NL, Kern KC, Giesser BS, Arshanapalli A, Schultz A, Montag M, et al. Regional hippocampal atrophy in multiple sclerosis. Brain 2008;131:1134-41.

36. Bishop CA, Newbould RD, Lee JS, Honeyfield L, Quest R, Colasanti A, et al. Analysis of ageingassociated grey matter volume in patients with multiple sclerosis shows excess atrophy in subcortical regions. Neurolmage Clinical 2017;13:9-15.

37. Blumcke I, Pauli E, Clusmann H, Schramm J, Becker A, Elger C, et al. A new clinico-pathological classification system for mesial temporal sclerosis. Acta Neuropathol 2007;113:235-44.

38. Mueller SG, Stables L, Du AT, Schuff N, Truran D, Cashdollar N, et al. Measurement of hippocampal subfields and age-related changes with high resolution MRI at 4T. Neurobiol Aging 2007;28:719-26.

39. Longoni G, Rocca MA, Pagani E, Riccitelli GC, Colombo B, Rodegher M, et al. Deficits in memory and visuospatial learning correlate with regional hippocampal atrophy in MS. Brain Struct Funct 2015;220:43544.

40. Planche V, Koubiyr I, Romero JE, Manjon JV, Coupe P, Deloire M, et al. Regional hippocampal vulnerability in early multiple sclerosis: Dynamic pathological spreading from dentate gyrus to CA1. Hum Brain Mapp 2018;39:1814-24.

41. Kiy G, Lehmann P, Hahn HK, Eling P, Kastrup A, Hildebrandt H. Decreased hippocampal volume, indirectly measured, is associated with depressive symptoms and consolidation deficits in multiple sclerosis. Mult Scler 2011;17:1088-97.

42. Gold SM, Kern KC, O'Connor MF, Montag MJ, Kim A, Yoo YS, et al. Smaller cornu ammonis 2$3 /$ dentate gyrus volumes and elevated cortisol in multiple sclerosis patients with depressive symptoms. Biol Psychiatry 2010;68:553-9.

43. Gold SM, O'Connor MF, Gill R, Kern KC, Shi Y, Henry RG, et al. Detection of altered hippocampal morphology in multiple sclerosis-associated depression using automated surface mesh modeling. Hum Brain Mapp 2014;35:30-7. 
44. Rocca MA, Longoni G, Pagani E, Boffa G, Colombo B, Rodegher M, et al. In vivo evidence of hippocampal dentate gyrus expansion in multiple sclerosis. Hum Brain Mapp 2015;36:4702-13.

45. Roosendaal SD, Hulst HE, Vrenken H, Feenstra HE, Castelijns JA, Pouwels PJ, et al. Structural and functional hippocampal changes in multiple sclerosis patients with intact memory function. Radiology 2010;255:595-604.

46. Gonzalez Torre JA, Cruz-Gomez AJ, Belenguer A, Sanchis-Segura C, Avila C, Forn C. Hippocampal dysfunction is associated with memory impairment in multiple sclerosis: A volumetric and functional connectivity study. Mult Scler 2017;23:1854-63.

47. Hulst HE, Schoonheim MM, Roosendaal SD, Popescu V, Schweren LJ, van der Werf YD, et al. Functional adaptive changes within the hippocampal memory system of patients with multiple sclerosis. Hum Brain Mapp 2012;33:2268-80.

48. Hulst HE, Schoonheim MM, Van Geest $Q$, Uitdehaag BM, Barkhof F, Geurts JJ. Memory impairment in multiple sclerosis: Relevance of hippocampal activation and hippocampal connectivity. Mult Scler 2015;21:1705-12.

49. Koenig KA, Rao SM, Lowe MJ, Lin J, Sakaie KE, Stone L, et al. The role of the thalamus and hippocampus in episodic memory performance in patients with multiple sclerosis. Mult Scler 2018:1352458518760716.

50. Rocca MA, Pravata E, Valsasina P, Radaelli M, Colombo B, Vacchi L, et al. Hippocampal-DMN disconnectivity in MS is related to WM lesions and depression. Hum Brain Mapp 2015;36:5051-63.

51. Colasanti A, Guo Q, Giannetti P, Wall MB, Newbould RD, Bishop C, et al. Hippocampal Neuroinflammation, Functional Connectivity, and Depressive Symptoms in Multiple Sclerosis. Biol Psychiatry 2016;80:62-72.

52. Firth J, Stubbs B, Vancampfort D, Schuch F, Lagopoulos J, Rosenbaum S, et al. Effect of aerobic exercise on hippocampal volume in humans: A systematic review and meta-analysis. Neuroimage 2018;166:230-8.

53. Erickson KI, Voss MW, Prakash RS, Basak C, Szabo A, Chaddock L, et al. Exercise training increases size of hippocampus and improves memory. Proc Natl Acad Sci U S A 2011;108:3017-22.

54. Erickson KI, Miller DL, Roecklein KA. The aging hippocampus: interactions between exercise, depression, and BDNF. Neuroscientist 2012;18:82-97.

55. Pajonk FG, Wobrock T, Gruber O, Scherk H, Berner D, Kaizl I, et al. Hippocampal plasticity in response to exercise in schizophrenia. Arch Gen Psychiatry 2010;67:133-43.

56. Hampstead BM, Stringer AY, Stilla RF, Giddens M, Sathian K. Mnemonic strategy training partially restores hippocampal activity in patients with mild cognitive impairment. Hippocampus 2012;22:1652-8.

57. Eriksson PS, Perfilieva E, Bjork-Eriksson T, Alborn AM, Nordborg C, Peterson DA, et al. Neurogenesis in the adult human hippocampus. Nature Medicine 1998;4:1313-7.

58. Spalding KL, Bergmann O, Alkass K, Bernard S, Salehpour M, Huttner HB, et al. Dynamics of hippocampal neurogenesis in adult humans. Cell 2013;153:1219-27.

59. Kempermann G, Song HJ, Gage FH. Neurogenesis in the Adult Hippocampus. Csh Perspect Biol 2015;7.

60. Frankland PW, Kohler S, Josselyn SA. Hippocampal neurogenesis and forgetting. Trends Neurosci 2013;36:497-503.

61. Zhao CM, Deng W, Gage FH. Mechanisms and functional implications of adult neurogenesis. Cell 2008;132:645-60.

62. Boldrini M, Fulmore CA, Tartt AN, Simeon LR, Pavlova I, Poposka V, et al. Human Hippocampal Neurogenesis Persists throughout Aging. Cell Stem Cell 2018;22:589-99 e5.

63. Sorrells SF, Paredes MF, Cebrian-Silla A, Sandoval K, Qi D, Kelley KW, et al. Human hippocampal neurogenesis drops sharply in children to undetectable levels in adults. Nature 2018;555:377-81.

64. Kempermann G, Gage FH, Aigner L, Song H, Curtis MA, Thuret S, et al. Human Adult Neurogenesis: Evidence and Remaining Questions. Cell Stem Cell 2018;5:25-30.

65. Rehfeld K, Muller P, Aye N, Schmicker M, Dordevic M, Kaufmann J, et al. Dancing or Fitness Sport? The Effects of Two Training Programs on Hippocampal Plasticity and Balance Abilities in Healthy Seniors. Front Hum Neurosci 2017;11:305. 
66. Rosen AC, Sugiura L, Kramer JH, Whitfield-Gabrieli S, Gabrieli JD. Cognitive training changes hippocampal function in mild cognitive impairment: a pilot study. J Alzheimers Dis 2011;26 Suppl 3:349-57. 67. Gauthier LV, Taub E, Perkins C, Ortmann M, Mark VW, Uswatte G. Remodeling the brain: plastic structural brain changes produced by different motor therapies after stroke. Stroke 2008;39:1520-5. 68. Suo C, Singh MF, Gates N, Wen W, Sachdev P, Brodaty H, et al. Therapeutically relevant structural and functional mechanisms triggered by physical and cognitive exercise. Mol Psychiatry 2016;21:1645.

69. Prosperini L, Piattella MC, Gianni C, Pantano P. Functional and Structural Brain Plasticity Enhanced by Motor and Cognitive Rehabilitation in Multiple Sclerosis. Neural Plast 2015;2015:481574.

70. Chiaravalloti ND, Wylie G, Leavitt V, DeLuca J. Increased cerebral activation after behavioral treatment for memory deficits in MS. J Neurol 2012;259:1337-46.

71. Leavitt VM, Wylie GR, Girgis PA, DeLuca J, Chiaravalloti ND. Increased functional connectivity within memory networks following memory rehabilitation in multiple sclerosis. Brain Imaging Behav 2014;8:394402.

72. Leavitt VM, Cirnigliaro C, Cohen A, Farag A, Brooks M, Wecht JM, et al. Aerobic exercise increases hippocampal volume and improves memory in multiple sclerosis: preliminary findings. Neurocase 2014;20:695-7.

73. Pluchino S, Muzio L, Imitola J, Deleidi M, Alfaro-Cervello C, Salani G, et al. Persistent inflammation alters the function of the endogenous brain stem cell compartment. Brain 2008;131:2564-78.

74. Monje ML, Toda H, Palmer TD. Inflammatory blockade restores adult hippocampal neurogenesis. Science 2003;302:1760-5.

75. Giannakopoulou A, Lyras GA, Grigoriadis N. Long-Term Effects of Autoimmune CNS Inflammation on Adult Hippocampal Neurogenesis. J Neurosci Res 2017;95:1446-58.

76. Stessin AM, Gursel DB, Schwartz A, Parashar B, Kulidzhanov FG, Sabbas AM, et al. FTY720, sphingosine 1-phosphate receptor modulator, selectively radioprotects hippocampal neural stem cells. Neuroscience Letters 2012;516:253-8.

77. Planche V, Panatier A, Hiba B, Ducourneau EG, Raffard G, Dubourdieu N, et al. Selective dentate gyrus disruption causes memory impairment at the early stage of experimental multiple sclerosis. Brain Behav Immun 2017;60:240-54.

78. Ciampi E, Pareto D, Sastre-Garriga J, Vidal-Jordana A, Tur C, Rio J, et al. Grey matter atrophy is associated with disability increase in natalizumab-treated patients. Mult Scler 2017;23:556-66.

79. Duvernoy HM. The Human Hippocampus: Functional Anatomy, Vascularization and Serial Sections with MRI. 5th ed ed. Berlin; 2005.

80. Poppenk J, Evensmoen HR, Moscovitch M, Nadel L. Long-axis specialization of the human hippocampus. Trends Cogn Sci 2013;17:230-40.

81. Fanselow MS, Dong HW. Are the dorsal and ventral hippocampus functionally distinct structures? Neuron 2010;65:7-19. 


\section{Figure legends}

\section{Figure 1. Schematic representation of hippocampal subdivisions and connections.}

Anatomically, the hippocampus consists of the cornu ammonis (CA), a heterogeneous structure divided into four histological subfields (CA1-CA4), and the DG. The CA and DG form two laminae rolled up one inside the other. The subiculum, which extends from the CA, is also part of the hippocampal formation. ${ }^{79}$ There is a further functional and anatomical subdivision of the hippocampus along its long-axis, ${ }^{80}$ with the posterior hippocampus involved primarily in cognitive functions and the anterior portion related to stress, emotion and affect. ${ }^{81}$ The posterior hippocampus forms a critical network with the retrosplenial and anterior cingulate cortical areas, inferior parietal cortex and the thalamus. The anterior hippocampus is connected with the amygdala, olfactory cortex, insula and ventromedial prefrontal cortex, helping to control emotional experience and the regulation of affective states. At the top of the figure (A) a model of long-axis hippocampal connectivity specialization is shown. Using BrainNet Viewer software starting from the automatic anatomic labeling (AAL) of the hippocampal region, a 3-dimensional reconstruction of left and right hippocampi is shown in magenta. A coronal section separates anterior and posterior hippocampal formations. Brain regions connected with the anterior and posterior hippocampi are respectively represented as spherical regions of interests in turquoise and dark blue. At the bottom of the figure (B), hippocampal subfields in a healthy subject, obtained by performing freesurfer high resolution automatic segmentation (http://surfer.nmr.mgh.harvard.edu/Freesurfer6.0.0), are shown in different orientation (axial, coronal and sagittal). To produce this graphical representation, hippocampal subfields masks from the freesurfer segmentation were overlaid on a T1-weighted MRI images using FSLeyes.

Abbreviations: IPL=inferior parietal lobule; $\mathrm{ACC}=$ anterior cingulate cortex; $\mathrm{VmPFC}=$ ventromedial prefrontal cortex. 
Figure 2. Hippocampal pathology in MS. Proteolipid protein staining (A) and schematic drawing (B) of a control hippocampus (coronally cut; original magnification: $2.5 \times$ ), showing various anatomical regions. I, molecular layer of the dentate gyrus (DG) (top: hilus, bottom: molecular layer of the DG); II, hilus of the DG; III, cornu ammonis (CA) 3; IV, CA2; V, CA1, superior part; VI, CA1, inferior part; VII, subiculum; VIII, parahippocampal gyrus. The arrow indicates the collateral sulcus, beyond which no grey matter or white matter (WM) lesions were scored. Large, mixed hippocampal-perihippocampal lesions in 2 representative cases with memory impairment (proteolipid protein stainings) are shown in C and D. (C) Mixed hippocampal-perihippocampal lesion (right black arrow) with a protruding WM part (white arrow) that contained Human Leukocyte Antigen (HLA)-antigen D Relate (DR)-positive reactive microglia at the border. The lesion covered CA1 and also a large part of the subiculum. An estimated 75\% of the myelin in CA3 was lost and the molecular layer of the DG was fully demyelinated (left black arrow). CA2 was relatively intact (only a slight decrease in myelin density was observed). (D) Large periventricular mixed lesion (white arrow) that extended into the hippocampus (black arrow). CA1, CA2, and part of CA3 were demyelinated. The hilus was intact, as well as the molecular layer of the DG. All samples were cut coronally (Zeiss Mirax scan; original magnification: 3x). Reproduced from Geurts et al., ${ }^{16}$ by permission of Oxford University Press.

\section{Figure 3. DIR sequences show more hippocampal lesions than conventional T2-weighted}

sequences. Coronal 3D double inversion recovery (upper rows) and T2-weighted (lower rows) images from two MS patients (A and B). In A, lesions in the left hippocampus (arrow) are visible on both sequences. In B, a hippocampal lesion is visible on the DIR sequence, but not on the T2weighted sequence. Reproduced from Roosendaal et al., ${ }^{24}$ by permission of John Wiley and Sons. 